\title{
A SYSTEMATIC DEVELOPMENT OF 'SOLID-SHELL' ELEMENT FORMULATIONS FOR LINEAR AND NON-LINEAR ANALYSES EMPLOYING ONLY DISPLACEMENT DEGREES OF FREEDOM
}

\author{
R. HAUPTMANN AND K. SCHWEIZERHOF* \\ Institute of Mechanics, University of Karlsruhe, Germany
}

\begin{abstract}
In the present contribution we propose a so-called solid-shell concept which incorporates only displacement degrees of freedom. Thus, some major disadvantages of the usually used degenerated shell concept are overcome. These disadvantages are related to boundary conditions - the handling of soft and hard support, the need for special co-ordinate systems at boundaries, the connection with continuum elements - and, in geometrically non-linear analyses, to a complicated update of the rotation vector.

First, the kinematics of the so-called solid-shell concept in analogy to the degenerated shell concept are introduced. Then several modifications of the solid-shell concept are proposed to obtain locking-free solidshell elements, leading also to formulations which allow the use of general three-dimensional material laws and which are also able to represent the normal stresses and strains in thickness direction. Numerical analyses of geometrically linear and non-linear problems are finally performed using solely assumed natural shear strain elements with a linear approximation in in-plane direction.

Although some considerations are needed to get comparable boundary conditions in the examples analysed, the solid-shell elements prove to work as good as the degenerated shell elements. The numerical examples show that neither thickness nor shear locking are present even for distorted element shapes. (C) 1998 John Wiley \& Sons, Ltd.
\end{abstract}

KEY WORDS: linear finite element analysis; geometrically non-linear finite element analysis; element technology; shell elements

\section{INTRODUCTION}

General shell structures contain flat, slightly curved and highly curved parts. They also consist of parts with two- or three-dimensional stress states. The analyses of their linear and non-linear behaviour should be accurate and efficient. It is therefore desirable to combine different element types, such that the calculation of each part is as efficient and as accurate as possible.

Most efficient shell elements are based on the degenerated shell concept (see e.g. References 1-3). The starting point of this shell concept is the kinematics of the three-dimensional continuum modified by the three following assumptions and resulting in a mid-surface description in

\footnotetext{
${ }^{*}$ Correspondence to: K. Schweizerhof, Institut fur Mechanik, Universitat Karlsruhe (TH), Kaiserstrasse 12, 76128 Karlsruhe, Germany. E-mail: mechanik@bau-verm.uni-karlsruhe.de

CCC 0029-5981/98/010049-21\$17.50

Received 14 November 1996

(C) 1998 John Wiley \& Sons, Ltd.

Revised 29 April 1997
} 
analogy to standard shell theory (see References 4 and 5). First, the normals to the mid-surface in the initial configuration remain straight but not normal during the deformation. Second, the thickness of the elements remains constant and third the normal stress in thickness direction is neglected. Although these approximations lead to very good results in most cases, there could arise difficulties concerning the rotational degrees of freedom. In particular, when describing the boundary conditions, special care of the nodal co-ordinate systems must be taken. Also there is always the question, how to handle the rotations normal to the boundary-soft or hard support. A complicated update of the rotations is necessary in geometrically non-linear situations. Additional problems arise when shell elements are used in combination with solid elements. Then special transition elements are necessary to combine shell elements having three displacement and two rotational degrees of freedom with solid elements containing six displacement degrees of freedom. Another difficulty occurs in using general three-dimensional material laws due to the requirement to reduce the material law according to the normal stress condition.

So far there are two promising approaches to avoid the disadvantages mentioned above.

The first one described by e.g. Sansour ${ }^{6}$ and Braun ${ }^{7}$ does not constrain the element thickness and takes the normal stress in thickness direction into account. Although these differences to the degenerated shell concept allow the use of general three-dimensional material laws without any further modification, there are seven instead of five degrees of freedom per node necessary to avoid locking. Special transition elements are necessary to combine these elements with solid elements even if a kinematics with a director difference vector ${ }^{7}$ is used which leads to a formulation with no rotational degrees of freedom.

The second one, well known as multidirector formulation and originally developed for the analysis of layered structures (see e.g. Reference 8) does not constrain the element thickness and takes the normal stress in thickness direction into account. However, the complex formulation with several layers and the ability to describe three-dimensional stress states may lead to an inefficient analysis, if these elements are used throughout the whole problem even for homogeneous isotropic cases. In the present contribution we propose in analogy to earlier developments by Hallquist ${ }^{9}$ a solid-shell concept which incorporates the kinematics of the three-dimensional continuum evaluated in a local co-ordinate system aligned to the upper and lower surface. Thus, the well-known assumed strain approach ${ }^{10}$ to avoid transversal shear locking can be applied and in addition the approximations of the displacements in thickness and in-plane direction can be performed seperately. The assumption that the normals remain straight is fulfilled by a linear approximation of the in-plane displacements over the thickness. By using a linear interpolation of the displacements in thickness direction in combination with a general three-dimensional material law, a locking effect due to the normal thickness stresses and strains occurs. To circumvent this problem several element formulations with different interpolations of the displacements resp. normal strains in thickness direction are developed in a consistent manner and discussed concerning their range of application. Two of the proposed solid-shell elements turn out to be rather similar to developments by Seifert ${ }^{11}$ and Parisch ${ }^{12}$ based on the kinematics presented by Schoop ${ }^{13}$ and an interpolation in thickness direction proposed by Büchter ${ }^{14}$ and Braun. ${ }^{7}$

The concluding numerical examples are chosen to demonstrate the capabilities of the solid-shell concept compared to the degenerated shell concept. There is also a focus on the three-dimensional features of the solid-shells and their condition numbers in particular in the case of rather thin shells. Finally, the behaviour of the solid-shells for large displacement/large rotation problems is shown compared to alternative solutions. 


\section{THE SOLID-SHELL CONCEPT}

In this section a shell concept is proposed that allows to overcome the known problems associated with the rotational degrees of freedom of degenerated shell elements. However, it is essential to achieve at least the good behaviour of the latter type elements and, optionally, to allow the usage of general three-dimensional material laws without any further modification. Therefore, the assumption of the degenerated shell concept that the normals to the element mid-surface remain straight but not necessarily normal during the deformation, is adopted. Thus, the initial threedimensional continuum of the shell geometry

$$
\mathbf{X}(\xi, \eta, \zeta)=\frac{1}{2}\left((1+\zeta) \mathbf{X}_{\mathrm{u}}(\xi, \eta)+(1-\zeta) \mathbf{X}_{\ell}(\xi, \eta)\right)
$$

and the displacements

$$
\mathbf{u}(\xi, \eta, \zeta)=\mathbf{T}(\xi, \eta) \Theta(\zeta)\left[\begin{array}{cc}
\mathbf{T}^{\mathrm{T}}(\xi, \eta) & \mathbf{0}_{3 \times 3} \\
\mathbf{0}_{3 \times 3} & \mathbf{T}^{\mathrm{T}}(\xi, \eta)
\end{array}\right]\left[\begin{array}{l}
\mathbf{u}_{\mathrm{u}}(\xi, \eta) \\
\mathbf{u}_{\ell}(\xi, \eta)
\end{array}\right]
$$

are approximated in global Cartesian co-ordinates $\left(\mathbf{e}_{1}, \mathbf{e}_{2}, \mathbf{e}_{3}\right)$ (Figure 1). In equation (2) the matrix $\Theta(\zeta)$ contains the interpolation of the displacements in thickness direction such that the assumption cited above is fulfilled:

$$
\boldsymbol{\Theta}(\zeta)=\frac{1}{2}\left[\begin{array}{cccccc}
1+\zeta & 0 & 0 & 1-\zeta & 0 & 0 \\
0 & 1+\zeta & 0 & 0 & 1-\zeta & 0 \\
0 & 0 & 1+\zeta & 0 & 0 & 1-\zeta
\end{array}\right]
$$

According to the degenerated shell concept $\xi$ and $\eta$ are local convective co-ordinates in in-plane direction and $\zeta$ is the local convective co-ordinate in thickness direction. The position $\mathbf{X}$ and the displacements $\mathbf{u}$ of each point of the shell is described by a position vector $\mathbf{X}_{\mathrm{u}}$ and displacements $\mathbf{u}_{\mathrm{u}}$ of the corresponding points on the upper shell surface and on the lower shell surface $\mathbf{X}_{\ell}$ and $\mathbf{u}_{\ell}$. In equation (2) $\mathbf{T}(\xi, \eta)$ is a transformation matrix from either a local Cartesian or a local convective co-ordinate system on the element mid-surface to the global co-ordinate system. Applying the relation $\mathbf{x}=\mathbf{X}+\mathbf{u}$ the geometry in the actual state which is essential for the evaluation of the deformation gradient can be computed.

Contrary to the kinematics used for degenerated shell elements the weak form

$$
\delta \Pi=\int_{V} \delta \mathbf{E} \cdot \mathbf{S} \mathrm{d} V+\delta \Pi_{\mathrm{ext}}=0 \quad \delta \Pi_{\mathrm{ext}} \quad \ldots \quad \text { external work }
$$

now contains the complete Green-Lagrange strain tensor $\mathbf{E}$ and the corresponding 2. PiolaKirchhoff stress tensor $\mathbf{S}$.

The kinematics of the solid-shell concept are identical to the kinematics of the 'Double-nodemodel' proposed by Schoop in $1986,{ }^{13}$ which is based directly on the degenerated shell concept leading to the geometry in the reference configuration

$$
\mathbf{X}(\xi, \eta, \zeta)=\underbrace{\frac{1}{2}\left(\mathbf{X}_{\mathrm{u}}(\xi, \eta)+\mathbf{X}_{\ell}(\xi, \eta)\right)}_{\mathbf{X}_{\mathrm{R}}(\xi, \eta)}+\frac{1}{2} \zeta \underbrace{\left(\mathbf{X}_{\mathrm{u}}(\xi, \eta)-\mathbf{X}_{\ell}(\xi, \eta)\right)}_{h(\xi, \eta) \mathbf{D}(\xi, \eta)}
$$




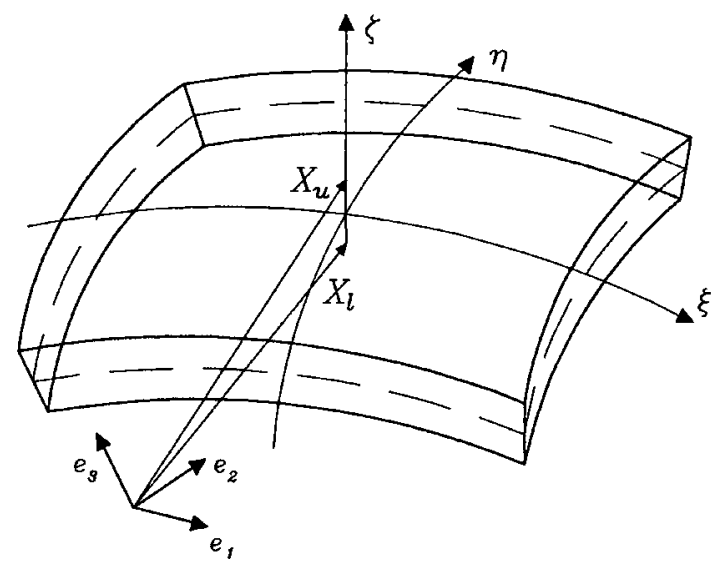

Figure 1. Geometry of the solid-shell element

and in the current configuration

$$
\mathbf{x}(\xi, \eta, \zeta)=\underbrace{\frac{1}{2}\left(\mathbf{x}_{\mathrm{u}}(\xi, \eta)+\mathbf{x}_{\ell}(\xi, \eta)\right)}_{\mathbf{x}_{\mathrm{R}}(\xi, \eta)}+\frac{1}{2} \zeta \underbrace{\left(\mathbf{x}_{\mathrm{u}}(\xi, \eta)-\mathbf{x}_{\ell}(\xi, \eta)\right)}_{h(\xi, \eta) \mathbf{d}(\xi, \eta)}
$$

with the thickness $h(\xi, \eta)$ and the director in the deformed $\mathbf{d}(\xi, \eta)$ and undeformed configuration $\mathbf{D}(\xi, \eta)$. It must be noted that in order to develop locking-free element formulations the solid-shell concept allows to change the interpolation in thickness direction by a simple modification of the interpolation matrix, see equation (3).

\subsection{Locking effects occurring to elements based on the solid-shell concept}

2.1.1. Transversal shear and membrane locking. In equivalence to degenerated shell elements solid-shell elements suffer from transversal shear and membrane locking. Several methods are known to circumvent this limitation, e.g. the Assumed Natural Strain Method (ANS), the Assumed Stress Method or the Enhanced Assumed Strain Method (EAS).

To apply one of these methods to solid-shell elements the quantities in the weak form in equation (4) have to be transformed to a local co-ordinate system with the two axes $\mathbf{X}^{\prime}, \mathbf{Y}^{\prime}$ aligned to the element mid-surface and the third axis $\mathbf{Z}^{\prime}$ in thickness direction.

2.1.2. Thickness locking. According to the investigations in the literature see e.g. References 6 and 7, a formulation with a linear $w^{\prime}$ displacement assumption in thickness direction tends to so-called thickness locking, which will be also observed in the numerical analyses. This undesired locking effect occurs due to the constant approximation of the strain $E_{\zeta \zeta}$ in thickness direction contrary to a linear $S_{\zeta \zeta}$ stress distribution found when bending occurs. The reason for the $E_{\zeta \zeta}$ varying linearly in thickness direction is the coupling between the linear in-plane strains and the normal stress in thickness direction if the Poisson-ratio is not equal to zero (see Figure 2).

This limitation could be overcome by applying the plane stress condition according to the degenerated shell concept or by directly assuming a linear distribution of the normal strain in 


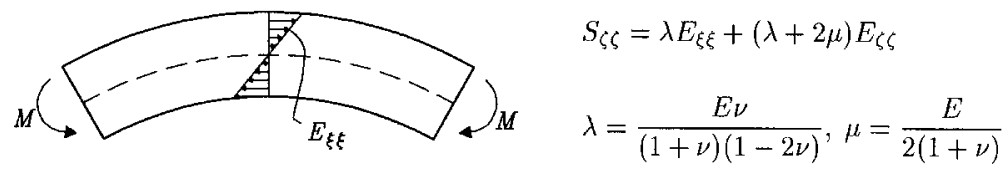

Figure 2. Beam subjected to bending moments

thickness direction over the thickness. As is well known, the plane stress assumption leads to a slightly too stiff behaviour in shell analyses.

\section{SYSTEMATIC DEVELOPMENT OF LOCKING-FREE SOLID-SHELL ELEMENTS}

\subsection{Solid-shell elements employing the plane stress condition}

This contribution is restricted to the linear elastic isotropic St. Venant-Kirchhoff material law. Modifying the material law according to the plane stress condition $\left(S_{\zeta \zeta}=0\right)$ leads to a linear displacement distribution in thickness direction. However, the corresponding weak form (4) does not contain any part for the thickness direction and therefore an element formulation results which exhibits zero energy modes.

To overcome this limitation several possibilities to fulfill the assumption of the degenerated shell concept that the element thickness remains constant during the deformation are investigated in the following. The first idea is to condense out one degree of freedom in equation (2) by the assumption that the displacement in thickness direction of the upper surface is equal to the displacement of the lower surface

$$
w^{\prime}(\xi, \eta, 1)=w^{\prime}(\xi, \eta,-1)
$$

which leads - contrary to the original solid-shell concept - to a formulation with five degrees of freedom. However, because of the constraint that the thickness direction must not be parallel to either the global $x$-axis or the global $y$-axis, this formulation lacks generality. Another approach is to add equation (7) by a penalty parameter to the equations resulting from (4). However, the resulting 'stiffness' matrix is then not symmetric and thus this approach is very inefficient.

Another formulation proposed in this contribution avoids zero energy modes by adding a stiffness in thickness direction. Therefore, the variational functional is modified in the following fashion:

$$
\begin{aligned}
\Pi & =\frac{1}{2} \int_{V} \mathbf{E} \cdot \mathbf{S} \mathrm{d} V+\frac{1}{2} \int_{V} E_{\zeta \zeta} \bar{E} E_{\zeta \zeta} \mathrm{d} V+\Pi_{\mathrm{ext}} \\
& =\frac{1}{2} \int_{V} \mathbf{E} \cdot \mathbf{C}_{\mathrm{red}} \mathbf{E} \mathrm{d} V+\frac{1}{2} \int_{V} E_{\zeta \zeta} \bar{E} E_{\zeta \zeta} \mathrm{d} V+\Pi_{\mathrm{ext}}
\end{aligned}
$$

with $E_{\zeta \zeta}$ being the Green-Lagrange strain component in thickness direction, $\mathbf{C}_{\text {red }}$ the modified St. Venant-Kirchhoff material matrix not including any value in $\zeta$ direction and $\bar{E}$ is Youngs modulus transformed to a local co-ordinate system. Thus, the first integral of the functional (8) contains in-plane and transversal shear strains and stresses. The second penalty-type integral term-with a penalty factor $\alpha=\bar{E}$-is constructed of the normal strain in thickness direction $E_{\zeta \zeta}$ evaluated from the displacements and the corresponding part of the normal stress $S_{\zeta \zeta}=\bar{E} E_{\zeta \zeta}$. Thus, normal stresses and strains in thickness direction can be computed. However, for the evaluation of these 


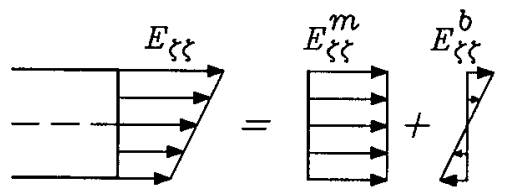

Figure 3. Division of the normal strains $E_{\zeta \zeta}$ into a constant and a linear part

stresses and strains the influence of the transversal contraction due to in-plane stretching is neglected. A minor but not better modification to this element formulation which is only mentioned for completeness is achieved by assuming a constant instead of linear $w^{\prime}$ displacement for the computation of the transversal shear strains $E_{\eta \zeta}$ and $E_{\zeta \zeta}$.

Another locking-free formulation with the additional advantage of obtaining a consistent thickness straining due to the coupling is obtained by dividing the normal stress in thickness direction into a constant - the membrane - and a linear - the bending - part (see Figure 3). After employing a one-point-integration in thickness direction for the coupling terms of the bending and membrane stresses and strains

$$
\int_{V} \mathbf{E}_{\mathrm{m}} \cdot \mathbf{S}_{\mathrm{b}} \mathrm{d} V=\int_{V} \mathbf{E}_{\mathrm{b}} \cdot \mathbf{S}_{\mathrm{m}} \mathrm{d} V=0
$$

the variational functional is written in the following form:

$$
\Pi=\underbrace{\frac{1}{2} \int_{V} \mathbf{E}_{\mathrm{b}} \cdot \mathbf{S}_{\mathrm{b}} \mathrm{d} V}_{\text {bending part }}+\underbrace{\frac{1}{2} \int_{V} \mathbf{E}_{\mathrm{m}} \cdot \mathbf{S}_{\mathrm{m}} \mathrm{d} V}_{\text {membrane part }}+\underbrace{\frac{1}{2} \int_{V} \mathbf{E}_{\mathrm{ts}} \cdot \mathbf{S}_{\mathrm{ts}} \mathrm{d} V}_{\text {shear part }}+\Pi_{\mathrm{ext}}
$$

To obtain a linear distribution of the normal strains in thickness direction, which is needed to avoid locking, again the zero normal stress condition according to the degenerated shell concept is applied to the bending part $S_{\zeta \zeta}^{\mathrm{b}}=0$. Thus, the linear normal strain in thickness direction

$$
E_{\zeta \zeta}^{\mathrm{b}}=-\frac{\lambda}{\lambda+2 \mu}\left(E_{\xi \xi}^{\mathrm{b}}+E_{\eta \eta}^{\mathrm{b}}\right)
$$

is introduced by a reduction of the St. Venant-Kirchhoff material law leading to the linear in-plane stresses $\mathbf{S}_{\mathrm{b}}=\mathbf{C}_{\mathrm{red}}^{\mathrm{ip}} \mathbf{E}_{\mathrm{b}}$. The constant in-plane stresses $\mathbf{S}_{\mathrm{m}}$ and the shear stresses $\mathbf{S}_{\mathrm{ts}}$ are computed using the general three-dimensional material law without any further modification. However, it must be noted that the assumed distributions of the normal stresses and strains in thickness direction over the thickness are only correct for flat elements. This effect will be visible for curved structures.

\subsection{Solid-shell elements employing directly a linear distribution of the normal strains in thickness direction}

One way to achieve a linear distribution of the normal stress in thickness direction is to assume a quadratic interpolation of the displacement $w^{\prime}$ in thickness direction as suggested by Gruttmann. ${ }^{8}$ By assuming a quadratic hierarchical distribution of the $w^{\prime}$ displacement (see Figure 4 ) the 


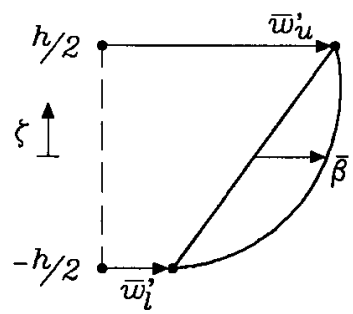

Figure 4. Hierarchical quadratic interpolation in $\zeta$ direction

displacements are approximated as follows:

$$
\mathbf{u}(\xi, \eta, \zeta)=\mathbf{T}(\xi, \eta) \overline{\mathbf{\Theta}}(\zeta)\left[\begin{array}{ccc}
\mathbf{T}^{\mathrm{T}}(\xi, \eta) & \mathbf{0}_{3 \times 3} & \mathbf{0}_{3 \times 1} \\
\mathbf{0}_{3 \times 3} & \mathbf{T}^{\mathrm{T}}(\xi, \eta) & \mathbf{0}_{3 \times 1} \\
\mathbf{0}_{1 \times 3} & \mathbf{0}_{1 \times 3} & 1
\end{array}\right]\left[\begin{array}{c}
\mathbf{u}_{\mathrm{u}}(\xi, \eta) \\
\mathbf{u}_{\ell}(\xi, \eta) \\
\beta(\xi, \eta)
\end{array}\right]
$$

with the interpolation matrix containing the contributions in thickness direction

$$
\overline{\mathbf{\Theta}}(\zeta)=\frac{1}{2}\left[\begin{array}{ccccccc}
1+\zeta & 0 & 0 & 1-\zeta & 0 & 0 & 0 \\
0 & 1+\zeta & 0 & 0 & 1-\zeta & 0 & 0 \\
0 & 0 & 1+\zeta & 0 & 0 & 1-\zeta & 1-\zeta^{2}
\end{array}\right]
$$

A rather similar approach was introduced by Büchter ${ }^{14}$ for degenerated shells and adopted to the 'Double-node-model' (6) by Seifert ${ }^{11}$ leading to the approximation of the geometry in the current configuration

$$
\mathbf{x}(\xi, \eta, \zeta)=\frac{1}{2}\left(\mathbf{x}_{\mathrm{u}}(\xi, \eta)+\mathbf{x}_{\ell}(\xi, \eta)\right)+\frac{1}{2}\left(\zeta+\lambda(\xi, \eta) \zeta^{2}\right)\left(\mathbf{x}_{\mathrm{u}}(\xi, \eta)-\mathbf{x}_{\ell}(\xi, \eta)\right)
$$

The choice of the quadratic interpolation in thickness direction $\zeta$ together with the kinematics of the 'Double-node-model'11 leads to an inconsistent displacement approximation in thickness direction due to the quadratic term not vanishing at the surfaces $\zeta=1$ and $\zeta=-1$ for $\lambda(\xi, \eta) \neq 0$, e.g.

$$
\mathbf{x}(\xi, \eta, \zeta=1)=\mathbf{x}_{\mathrm{u}}(\xi, \eta)+\frac{1}{2} \lambda(\xi, \eta)\left(\mathbf{x}_{\mathrm{u}}(\xi, \eta)-\mathbf{x}_{\ell}(\xi, \eta)\right) \neq \mathbf{x}_{\mathrm{u}}(\xi, \eta)
$$

Also the element formulation proposed by Parisch ${ }^{12}$ is based on this geometry approximation, but the interpolation $1-\zeta^{2}$ instead of $\zeta^{2}$ in direction of the deformed director is used, leading to a consistent displacement approximation in thickness direction. The differences between the solidshell concept and the geometry approximation in equation (14) are obvious if the displacements are written in local co-ordinates aligned to the shell mid-surface

$$
\mathbf{u}^{\prime}(\xi, \eta, \zeta)=\frac{1}{2}\left(\mathbf{u}_{\mathrm{u}}^{\prime}(\xi, \eta)+\mathbf{u}_{\ell}^{\prime}(\xi, \eta)\right)+\frac{1}{2} \zeta\left(\mathbf{u}_{\mathrm{u}}^{\prime}(\xi, \eta)-\mathbf{u}_{\ell}^{\prime}(\xi, \eta)\right)+\mathbf{h}(\xi, \eta)
$$

In equation (16) the abbreviation $\mathbf{h}(\xi, \eta)$ takes for the solid-shell concept the form

$$
\mathbf{h}(\xi, \eta)=\left(\begin{array}{l}
h_{x}^{\prime} \\
h_{y}^{\prime} \\
h_{z}^{\prime}
\end{array}\right)=\frac{1}{2}\left(1+\zeta^{2}\right) \beta(\xi, \eta)\left(\begin{array}{l}
0 \\
0 \\
1
\end{array}\right)
$$


and for the geometry approximation according to Seifert and Parisch the form

$$
\mathbf{h}(\xi, \eta)=\left(\begin{array}{c}
h_{x}^{\prime} \\
h_{y}^{\prime} \\
h_{z}^{\prime}
\end{array}\right)=\frac{1}{2} \hat{f}(\zeta) \lambda(\xi, \eta)\left(\begin{array}{c}
u_{\mathrm{u}}^{\prime}-u_{\ell}^{\prime} \\
v_{\mathrm{u}}^{\prime}-v_{\ell}^{\prime} \\
w_{\mathrm{u}}^{\prime}-w_{\ell}^{\prime}
\end{array}\right) \quad \text { with } \hat{f}(\zeta)= \begin{cases}\zeta^{2} & \text { Seifert } \\
1-\zeta^{2} & \text { Parisch }\end{cases}
$$

Obviously, the interpolation of Seifert and Parisch may lead to a quadratic distribution of the in-plane strains and therefore a mild locking may occur in transversal shear dominated cases. In addition, Parisch eliminates the seventh degree of freedom per edge on element level by static condensation resulting in thickness strains which are inconsistent to the neighbour elements in in-plane direction. Such a condensation is rather complicated for a consistent treatment in geometrically non-linear problems and similar to the procedure described in Box 1 .

1. Update on element level

- Nodal displacements

$$
\mathbf{d}_{\mathrm{e}}^{k+1}=\mathbf{d}_{\mathrm{e}}^{k}+\Delta \mathbf{d}_{\mathrm{e}}^{k}
$$

- Element parameters

$$
\alpha_{\mathrm{e}}^{k+1}=\alpha_{\mathrm{e}}^{k}-\left(\mathbf{D}_{\mathrm{e}}\right)^{-1}\left(\mathbf{L}_{\mathrm{e}}^{k} \Delta \mathbf{d}_{\mathrm{e}}^{k}+\mathbf{P}_{\mathrm{e}}^{k}\right)
$$

2. Computation on element level and at each integration point

- Enhanced strains

$$
\tilde{\mathbf{E}}=\mathbf{M}_{\mathrm{e}} \alpha_{\mathrm{e}}^{k+1}
$$

- Tangent stiffness matrix

$$
\mathbf{K}_{\text {tang }}^{\mathrm{e}}=\mathbf{K}_{\mathrm{e}}-\mathbf{L}_{\mathrm{e}}^{\mathrm{T}} \mathbf{D}_{\mathrm{e}}^{-1} \mathbf{L}_{\mathrm{e}}
$$

- Element residual vector

$$
\mathbf{f}^{\mathrm{e}}=\mathbf{f}_{\mathrm{ext}}^{\mathrm{e}}-\mathbf{R}_{\mathrm{e}}+\mathbf{L}_{\mathrm{e}}^{\mathrm{T}} \mathbf{D}_{\mathrm{e}}^{-1} \mathbf{P}_{\mathrm{e}}
$$

3. Global computation

- Assembly of the global matrices/vectors

- Solution of the global system of equations

$$
\Delta \mathbf{d}^{k+1}=\left(\mathbf{K}_{\text {tang }}^{k+1}\right)^{-1} \mathbf{f}^{k+1}
$$

- Convergence control:

$-\left\|\mathbf{f}^{k+1}\right\|<$ Tol. $\leadsto$ next load step

$-\left\|\mathbf{f}^{k+1}\right\|>$ Tol. $\rightsquigarrow k=k+1$ and go to 1 .

Box 1. Iterative solution algorithm for non-linear problems

Another way to obtain a linear distribution of the normal strain in thickness direction could be achieved by adding to the Green-Lagrange strain $E_{\zeta \zeta}^{k}$ a component $\tilde{E}_{\zeta \zeta}$ with a linear variation in 
thickness direction

$$
E_{\zeta \zeta}=E_{\zeta \zeta}^{k}+\tilde{E}_{\zeta \zeta}
$$

in analogy to the enhanced assumed strain method (EAS). ${ }^{15}$ This method is based on the wellknown three-field functional of $\mathrm{Hu}-\mathrm{Washizu}$ which can be written as two-field functional

$$
\Pi_{\mathrm{HW}}(\mathbf{u}, \tilde{\mathbf{E}})=\int_{V} W\left(\mathbf{E}_{k}+\tilde{\mathbf{E}}\right) \mathrm{d} V+\Pi_{\text {ext }}(\mathbf{u}) \quad W \quad \ldots \quad \text { Energy density function }
$$

by employing the well-known assumptions of the EAS method. Also the corresponding weak form

$$
\delta \Pi_{\mathrm{HW}}(\mathbf{u}, \tilde{\mathbf{E}})=\int_{V}\left(\delta \mathbf{E}_{k}+\delta \tilde{\mathbf{E}}\right) \cdot \mathbf{S} \mathrm{d} V+\delta \Pi_{\text {ext }}(\mathbf{u}) \quad \text { with } \quad \mathbf{S}=\frac{\partial W}{\partial \mathbf{E}}
$$

is obtained.

Discretizing the displacements $\mathbf{u}=\mathbf{u}\left(\mathbf{d}_{\mathrm{e}}\right)$ where $\mathbf{d}_{\mathrm{e}}$ is the element nodal displacement vector and discretizing also the assumed strains $\tilde{\mathbf{E}}=\tilde{\mathbf{E}}\left(\alpha_{\mathrm{e}}\right)$ where $\alpha_{\mathrm{e}}$ are independent parameters on element level the following tangent stiffness matrix:

$$
\mathbf{K}_{\text {tang }}^{\mathrm{e}}=\mathbf{K}_{\mathrm{e}}-\mathbf{L}_{\mathrm{e}}^{\mathrm{T}} \mathbf{D}_{\mathrm{e}}^{-1} \mathbf{L}_{\mathrm{e}}
$$

and the corresponding load vector

$$
\mathbf{f}^{\mathrm{e}}=\mathbf{f}_{\mathrm{ext}}^{\mathrm{e}}-\mathbf{f}_{\text {int }}^{\mathrm{e}}=\mathbf{f}_{\mathrm{ext}}^{\mathrm{e}}-\left(\mathbf{R}_{\mathrm{e}}-\mathbf{L}_{\mathrm{e}}^{\mathrm{T}} \mathbf{D}_{\mathrm{e}}^{-1} \mathbf{P}_{\mathrm{e}}\right)
$$

are obtained by using the abbreviations

$$
\begin{array}{ll}
\mathbf{K}_{\mathrm{e}}=\int_{V}\left(\frac{\partial^{2} \mathbf{E}_{k}}{\partial \mathbf{d}_{\mathrm{e}}^{2}} \cdot \mathbf{S}+\frac{\partial \mathbf{E}_{k}}{\partial \mathbf{d}_{\mathrm{e}}} \cdot \frac{\partial \mathbf{S}}{\partial \mathbf{d}_{\mathrm{e}}}\right) \mathrm{d} V & \mathbf{L}_{\mathrm{e}}=\int_{V} \frac{\partial \tilde{\mathbf{E}}}{\partial \boldsymbol{\alpha}_{\mathrm{e}}} \cdot \frac{\partial \mathbf{S}}{\partial \mathbf{d}_{\mathrm{e}}} \mathrm{d} V \\
\mathbf{R}_{\mathrm{e}}=\int_{V} \frac{\partial \mathbf{E}_{k}}{\partial \mathbf{d}_{\mathrm{e}}} \cdot \mathbf{S} \mathrm{d} V & \mathbf{P}_{\mathrm{e}}=\int_{V} \frac{\partial \tilde{\mathbf{E}}}{\partial \boldsymbol{\alpha}_{\mathrm{e}}} \cdot \mathbf{S} \mathrm{d} V \\
\mathbf{D}_{\mathrm{e}}=\int_{V} \frac{\partial \tilde{\mathbf{E}}}{\partial \boldsymbol{\alpha}_{\mathrm{e}}} \cdot \frac{\partial \mathbf{S}}{\partial \boldsymbol{\alpha}_{\mathrm{e}}} \mathrm{d} V &
\end{array}
$$

According to equation (19) the assumed strain field $\tilde{\mathbf{E}}(\alpha)$ contains solely zero elements except for

$$
\tilde{E}_{\zeta \zeta}=\zeta \mathbf{M}_{\mathrm{e}}(\xi, \eta) \alpha_{\mathrm{e}}
$$

with the matrix $\mathbf{M}_{\mathrm{e}}(\xi, \eta)$ describing the interpolation in in-plane direction which is chosen similar to the corresponding displacement interpolation. In addition, the matrix $\mathbf{M}_{\mathrm{e}}(\xi, \eta)$ generally contains a transformation to a co-ordinate system at the element midpoint in order to obtain a unique definition for the parameters $\alpha_{\mathrm{e}}$ throughout the whole element.

In Box 1 the solution procedure for the non-linear problem is summarized.

\section{FE DISCRETIZATION OF THE VARIOUS SOLID-SHELL ELEMENTS}

After discretization the geometry in the reference configuration (Figure 5)

$$
\mathbf{X}(\xi, \eta, \zeta)=\sum_{i=1}^{n} \frac{1}{2} N_{i}(\xi, \eta)\left((1+\zeta) \overline{\mathbf{X}}_{i \mathrm{u}}+(1-\zeta) \overline{\mathbf{X}}_{i \ell}\right)
$$




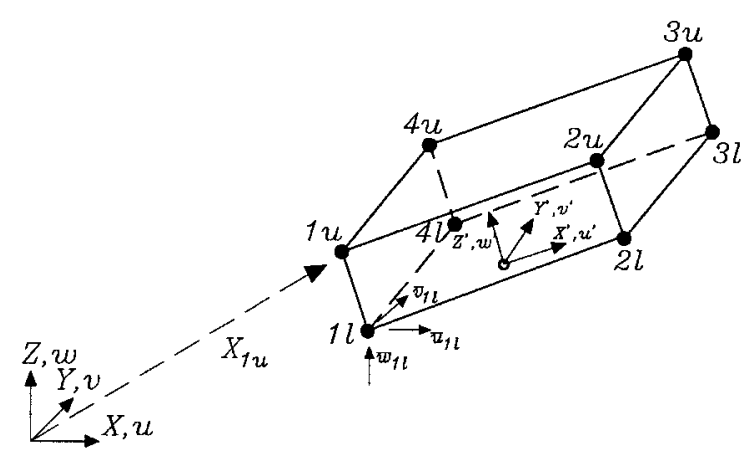

Figure 5. Discrete geometry of a solid-shell element

and the displacements

$$
\mathbf{u}(\xi, \eta, \zeta)=\sum_{i=1}^{n}\left(N_{i}(\xi, \eta) \mathbf{T}(\xi, \eta) \Theta(\zeta)\left[\begin{array}{cc}
\mathbf{T}^{\mathrm{T}}(\xi, \eta) & \mathbf{0}_{3 \times 3} \\
\mathbf{0}_{3 \times 3} & \mathbf{T}^{\mathrm{T}}(\xi, \eta)
\end{array}\right] \mathbf{d}_{\mathrm{e}}^{i}\right)
$$

depend on the two-dimensional shape functions in in-plane direction $N_{i}(\xi, \eta)$, on the element nodal displacement vector

$$
\mathbf{d}_{\mathrm{e}}^{i}=\left[\overline{\mathbf{u}}_{i \mathrm{u}}^{\mathrm{T}}, \overline{\mathbf{u}}_{i \ell}^{\mathrm{T}}\right]^{\mathrm{T}}=\left[\bar{u}_{i \mathrm{u}}, \bar{v}_{i \mathrm{u}}, \bar{w}_{i \mathrm{u}}, \bar{u}_{i \ell}, \bar{v}_{i \ell}, \bar{w}_{i \ell}\right]^{\mathrm{T}}
$$

and on the position vectors to the upper and lower node of edge $i$

$$
\overline{\mathbf{X}}_{i \mathrm{u}}=\left[\bar{X}_{i}, \bar{Y}_{i}, \bar{Z}_{i}\right]_{\mathrm{u}}^{\mathrm{T}} \quad \overline{\mathbf{X}}_{i \ell}=\left[\bar{X}_{i}, \bar{Y}_{i}, \bar{Z}_{i}\right]_{\ell}^{\mathrm{T}}
$$

In equations (26) and (27) $n$ denotes the number of edges per element and a bar stands for a displacement or position of a node of the corresponding edge $i$.

Choosing a local contravariant base system leads to the transformation matrix

$$
\mathbf{T}(\xi, \eta)=\left[\frac{\partial \mathbf{x}_{\mathrm{m}}(\xi, \eta)}{\partial \xi}, \frac{\partial \mathbf{x}_{\mathrm{m}}(\xi, \eta)}{\partial \eta}, \frac{\partial \mathbf{x}_{\mathrm{m}}(\xi, \eta)}{\partial \zeta}\right]
$$

with the defomed geometry of the middle surface

$$
\begin{aligned}
\mathbf{x}_{\mathrm{m}}= & \frac{1}{2}\left(\mathbf{x}_{\mathrm{u}}(\xi, \eta)+\mathbf{x}_{\ell}(\xi, \eta)\right) \\
& \frac{1}{2}\left(\mathbf{X}_{\mathrm{u}}(\xi, \eta)+\mathbf{u}_{\mathrm{u}}(\xi, \eta)+\mathbf{X}_{\ell}(\xi, \eta) \mathbf{u}_{\ell}(\xi, \eta)\right)
\end{aligned}
$$

Due to the relationship for linear problems

$$
\mathbf{T}(\xi, \eta)=\mathbf{J}^{\mathrm{T}}(\xi, \eta, \zeta=0) \quad \mathbf{J}(\xi, \eta, \zeta) \quad \ldots \quad \text { Jacobian matrix }
$$

this choice leads to a quite efficient element formulation. However, if the matrix (3) is not modified there is no need for a transformation and thus

$$
\mathbf{T}(\xi, \eta)=\mathbf{1}_{3 \times 3}
$$



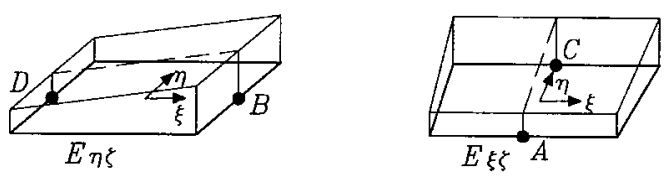

Figure 6. Assumed natural transversal shear strains

\subsection{Common aspects of the discretization of the investigated solid-shell elements}

Although the locking-free solid-shell elements proposed in Section 3 could be used with any order of approximation in in-plane direction, only elements with bilinear approximation are investigated in this contribution. Thus, the problem of transversal shear locking which appears with low-order Reissner-Mindlin elements has to be considered. In the case of the 4-node degenerated shell element, the assumed natural strain concept (ANS) developed by Bathe and Dvorkin ${ }^{16}$ has proven to be very efficient, very robust and gives very good results.

Thus, this method is combined with the element formulations proposed in the previous sections which is possible because the evaluation of the stresses and strains is performed in local co-ordinates. The essential assumption of the assumed natural strain concept is to assume the transversal shear interpolation in local convective co-ordinates to be constant in $\xi$ and linear in $\eta$ direction for $E_{\xi \zeta}$, respectively, to be constant in $\eta$ and linear in $\xi$ direction for $E_{\eta \zeta}$ (Figure 6)

$$
E_{\xi \zeta}=\frac{1}{2}(1-\eta) E_{\xi \zeta}^{\mathrm{A}}+\frac{1}{2}(1+\eta) E_{\xi \zeta}^{\mathrm{C}} \quad E_{\eta \zeta}=\frac{1}{2}(1-\xi) E_{\eta \zeta}^{\mathrm{D}}+\frac{1}{2}(1+\xi) E_{\eta \zeta}^{\mathrm{B}}
$$

The simplest element based on the solid-shell kinematics without any further modification besides using the interpolation of the transversal shear strains (34) has four edges per element, six degrees of freedom per edge and is named ANS3DL in the following sections.

\subsection{Solid-shell element formulations employing the plane stress assumption}

4.2.1. The ANS6z solid-shell element. The locking-free solid-shell element formulation named ANS6z is based on the modified functional (8). Due to the assumed strains (34) in local convective co-ordinates, a very efficient element formulation is obtained by evaluating both integrals of equation (8) in local convective co-ordinates. Therefore, the St. Venant-Kirchhoff material law

$$
C^{a b c d}=\lambda \delta^{a b} \delta^{c d}+\mu\left(\delta^{a c} \delta^{b d}+\delta^{a d} \delta^{b c}\right), \quad \delta^{i j}= \begin{cases}1, & i=j \\ 0, & i \neq j\end{cases}
$$

must be first transformed to local convective co-ordinates and then reduced according to the zero stress condition. In addition, the factor $\bar{E}$ is obtained from Youngs modulus $E$ by the following transformation:

$$
\bar{E}=\left(\frac{\partial \mathbf{X}}{\partial \zeta} \cdot \frac{\partial \mathbf{X}}{\partial \zeta}\right)\left(\frac{\partial \mathbf{X}}{\partial \zeta} \cdot \frac{\partial \mathbf{X}}{\partial \zeta}\right) E
$$

4.2.2. The ANS3DLr solid-shell element. Alternatively to the ANS6z element a solid-shell element formulation named ANS3DLr is introduced which takes the influence of the transversal contraction due to membrane stretching into account. Thus, this element is based on functional (10) 
which leads to the following weak form:

$$
\delta \Pi=\int_{V} \delta \mathbf{E}_{\mathrm{b}} \cdot \mathbf{C}_{\text {red }}^{\mathrm{ip}} \mathbf{E}_{\mathrm{b}} \mathrm{d} V+\int_{V} \delta \mathbf{E}_{\mathrm{m}} \cdot \mathbf{C}^{\mathrm{ip}} \mathbf{E}_{\mathrm{m}} \mathrm{d} V+\int_{V} \delta \mathbf{E}_{\mathrm{ts}} \cdot \mathbf{C}^{\mathrm{ts}} \mathbf{E}_{\mathrm{ts}} \mathrm{d} V+\Pi_{\text {ext }}
$$

In equation (37) the Green-Lagrange strains are split into the second-order tensor $\mathbf{E}_{\mathrm{b}}$ with in-plane strains varying linearly in thickness direction, the tensor $\mathbf{E}_{\mathrm{m}}$ with in-plane and normal strains constant in thickness direction and the tensor $\mathbf{E}_{\mathrm{ts}}$ of the transversal shear strain components. Then the fourth-order tensor $\mathbf{C}_{\text {red }}^{\text {ip }}$ contains the in-plane and thickness components of the reduced St. VenantKirchhoff material tensor, the tensors $\mathbf{C}^{\mathrm{ip}}$ and $\mathbf{C}^{\mathrm{ts}}$ contain the in-plane and thickness components, respectively, transversal shear components of the corresponding unmodified material tensor.

Again an evaluation of all three integrals of equation (37) in local convective co-ordinates results in a very efficient element formulation.

\subsection{Solid-shell element formulations employing directly a linear distribution of the normal strains in thicknesss direction}

4.3.1. The ANS3Dq solid-shell element. The first solid-shell element formulation named ANS3Dq which allows the use of general three-dimensional material laws is based on a hierarchical quadratic interpolation of the displacement $w^{\prime}$ in thickness direction. ${ }^{8}$ After discretization the displacement interpolation (12) takes the form

$$
\mathbf{u}(\xi, \eta, \zeta)=\sum_{i=1}^{4}\left(N_{i}(\xi, \eta) \mathbf{T}(\xi, \eta) \overline{\boldsymbol{\Theta}}(\zeta)\left[\begin{array}{ccc}
\mathbf{T}^{\mathrm{T}}(\xi, \eta) & \mathbf{0}_{3 \times 3} & \mathbf{0}_{3 \times 1} \\
\mathbf{0}_{3 \times 3} & \mathbf{T}^{\mathrm{T}}(\xi, \eta) & \mathbf{0}_{3 \times 1} \\
\mathbf{0}_{1 \times 3} & \mathbf{0}_{1 \times 3} & 1
\end{array}\right] \mathbf{d}_{\mathrm{e}}^{i}\right)
$$

with the matrix $\overline{\boldsymbol{\Theta}}(\zeta)$ containing the interpolation of the displacements in thickness direction (13) and the element nodal displacement vector

$$
\mathbf{d}_{\mathrm{e}}^{i}=\left[\overline{\mathbf{u}}_{i \mathrm{u}}^{\mathrm{T}}, \overline{\mathbf{u}}_{i \ell}^{\mathrm{T}}, \beta_{i}\right]^{\mathrm{T}}=\left[\bar{u}_{i \mathrm{u}}, \bar{v}_{i \mathrm{u}}, \bar{w}_{i \mathrm{u}}, \bar{u}_{i \ell}, \bar{v}_{i \ell}, \bar{w}_{i \ell}, \beta_{i}\right]^{\mathrm{T}} .
$$

Thus, the ANS3Dq solid-shell element formulation has seven instead of six degrees of freedom per edge $i$. Again the transformation matrix $\mathbf{T}$ is evaluated using equation (30) which is based on a linear interpolation of the displacements in thickness direction.

4.3.2. The ANS3DEAS solid-shell element. The solid-shell formulation introduced in this section is based on the two-field functional (20) and is named ANS3DEAS. Due to the bilinear displacement approximation in in-plane direction the following matrix:

$$
\mathbf{M}_{\mathrm{e}}=\left[\begin{array}{llll}
1 & \xi & \eta & \xi
\end{array}\right]
$$

first proposed by Büchter ${ }^{14}$ and the vector $\alpha_{\mathrm{e}}$ containing the four unknown element parameters

$$
\alpha_{\mathrm{e}}=\left[\begin{array}{llll}
\alpha_{1} & \alpha_{2} & \alpha_{3} & \alpha_{4}
\end{array}\right]^{\mathrm{T}}
$$

which are belonging to the enhanced normal strains (25), are chosen. In most cases a shell element based on a bilinear geometry and displacement approximation is flat and therefore the transformation of the parameters $\alpha_{\mathrm{e}}$ is neglected. 
Table I. Eigenvalues of a square element; thickness to length ratio $t / l=2 / 100$

\begin{tabular}{lcccccc}
\hline & \multirow{2}{*}{$\begin{array}{c}\text { Rigid body } \\
\text { motion }\end{array}$} & Kinematics & $<0 \cdot 1$ & $<1 \cdot 0$ & $<1000$ & Max. \\
\cline { 4 - 7 } & $1-6$ & - & $7-11$ & $12-17$ & $18-20$ & $2 \cdot 54$ \\
ANS5 & $1-6$ & - & $7-9$ & $10-16$ & $17-23$ & 1365 \\
ANS6z & $1-6$ & - & $7-9$ & $10-16$ & $17-23$ & 1838 \\
ANS3DLr & $1-6$ & - & 7 & $8-16$ & $17-23$ & 1838 \\
ANS3DL & $1-6$ & - & $7-9$ & $10-16$ & $17-26$ & 1838 \\
ANS3Dq & $1-6$ & - & $7-9$ & $10-16$ & $17-23$ & 1838 \\
ANS3DEAS & & - & & & & \\
\hline
\end{tabular}

Also it is straightforward to enhance the membrane strains following Reference 15 which leads to a superiour behaviour in membrane dominated cases. However, to limit the length of this contribution such a modification to the ANS3DEAS element is not discussed here but it is referred to Hauptmann. ${ }^{17}$

\section{NUMERICAL EXAMPLES}

The following numerical analyses are all performed with 4-edge, respectively, 8-node elements, based on the formulations described in the previous sections. In addition to the ANS6z, ANS3DL, ANS3DLr, ANS3Dq and ANS3DEAS elements, a 4-node element based on the degenerated shell concept and modified according to the assumed natural shear strain method is used for comparison purposes. This element is referred to as ANS5, see e.g. Gebhardt Reference 18.

\subsection{Investigation of eigenvalues}

To get some information about the element behaviour concerning zero energy modes and possible locking tendencies, the eigenvalues of each element are computed. The chosen element shape is typical for an element contained in a regular mesh of a square plate.

Two situations are investigated. First, an element with a thickness to length ratio of $t / l=2 / 100$ is chosen. This represents a discretization with very coarse meshes or a rather thin plate/shell. Then an element with a thickness to length ratio of $t / l=1$ is chosen. Such element shapes are necessary to obtain converged results in the case of highly curved structures e.g. the pinched cylinder (see Section 5.2) or at locations with almost three-dimensional stress states. The essential conclusion from the results listed in Tables I and II is that none of the element formulations shows a serious tendency to lock or exhibits zero energy modes. Also, it is obvious that all solid-shell formulations proposed in this contribution show in the case of very coarse meshes much larger eigenvalues than the elements based on the degenerated shell concept which is due to the large stiffness in thickness direction and a conditioning problem may result. However, if finer meshesmostly necessary for converged results - are considered, the difference between the eigenvalues of each element formulation is not significant. Remarkably, in the case of very fine meshes as e.g. needed to analyse highly curved structures, the eigenvalues of the degenerated shell concept are even larger than those of the solid-shells. 
Table II. Eigenvalues of a square element; thickness to length ratio $t / l=1$

\begin{tabular}{lccccc}
\hline & Rigid body & & \multicolumn{3}{c}{ Eigenvalues } \\
\cline { 5 - 6 } Element & motion & Kinematics & $<0 \cdot 1$ & $<1 \cdot 0$ & Max. \\
\hline ANS5 & $1-6$ & - & $7-12$ & $13-18$ & $1 \cdot 56$ \\
ANS6z & $1-6$ & - & $7-11$ & $12-24$ & $0 \cdot 78$ \\
ANS3DLr & $1-6$ & - & $7-10$ & $11-23$ & $1 \cdot 36$ \\
ANS3DL & $1-6$ & - & $7-8$ & $9-23$ & $1 \cdot 36$ \\
ANS3Dq & $1-6$ & - & $7-11$ & $12-27$ & $1 \cdot 36$ \\
ANS3DEAS & $1-6$ & - & $7-10$ & $11-23$ & $1 \cdot 36$ \\
\hline
\end{tabular}

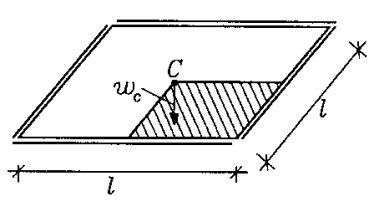

$$
\begin{aligned}
l & =10 \\
t & =0.1 \\
E & =10.92 \\
\nu & =0.3 \\
q & =1 \text { per area }
\end{aligned}
$$

Figure 7. Simply supported square plate

\subsection{Linear analyses}

5.2.1. Simply supported square plate under distributed loading. Due to the symmetry of the square plate (Figure 7) which is simply supported (hard), only one fourth of the plate is analyzed using one element in thickness direction. In the following three diagrams the normalized vertical displacement of the centre of the plate is shown over the number of elements per area. To normalize the vertical displacement the analytical solution of Timoshenko ${ }^{19}$ is used. The chosen thickness to length ratio accounts for a rather thin plate and locking should be observed, if present.

Because of the flat structure the vertical displacements of the upper surface are identical to those of the lower surface and the ANS5, ANS6z, ANS3DLr and ANS3DEAS elements lead to identical results. It is obvious that contrary to the other element formulations, the ANS3DL element does not converge to the analytical solution. It shows clearly a tendency to lock as mentioned before in Section 2.1.2 which can be attributed to the coupling of the normal stress in thickness direction and the strains in in-plane direction. To investigate this undesirable effect further, a discretization of the plate with $8 \times 8$ elements in in-plane direction is used while the number of elements in thickness direction is varied (Figure 9).

As shown in Figure 9 the modified solid-shell elements reproduce the analytical solution already with one element in thickness direction whereas eight elements are necessary for the ANS3DL formulation. It is also interesting to note that the three-dimensional effect is also visible for the ANS3Dq element, when eight elements are used in thickness direction.

The last important aspect is the influence of element distortions on the convergence properties. Thus, the vertical displacement of the distorted mesh shown in Figure 10 is computed for a discretization with varying mesh density.

For coarse meshes the results given in Figure 11 are slightly worse compared to the results of a regular mesh, but the overall behaviour of the elements remains unchanged. 


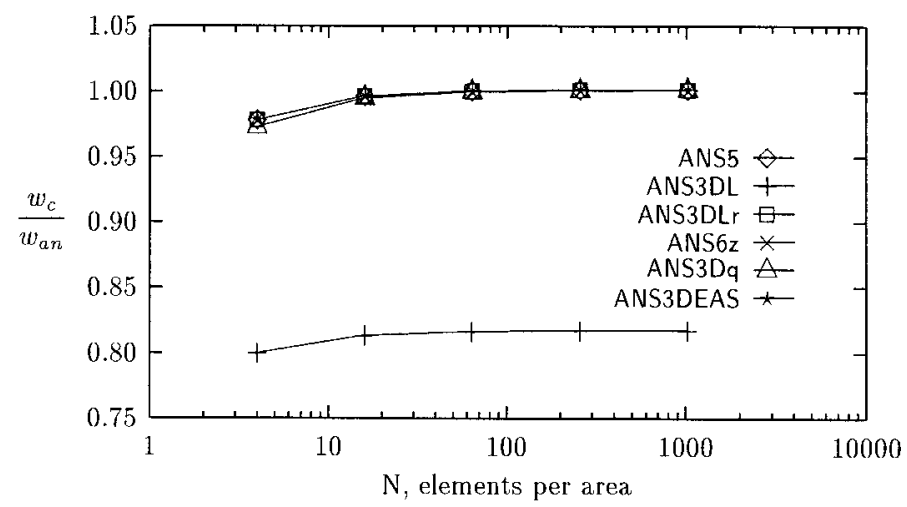

Figure 8. Convergence investigation for various element formulations; simply supported square plate with regular mesh

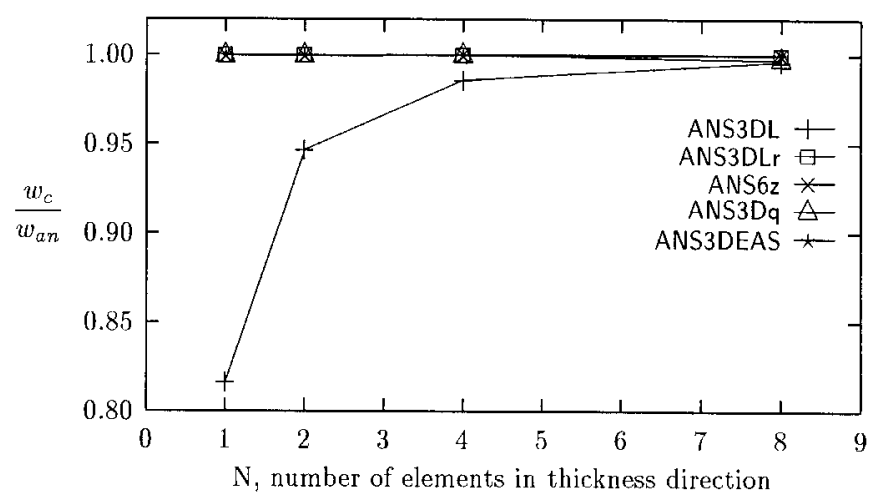

Figure 9. Convergence investigation for various element formulations; varying the number of elements in thickness direction

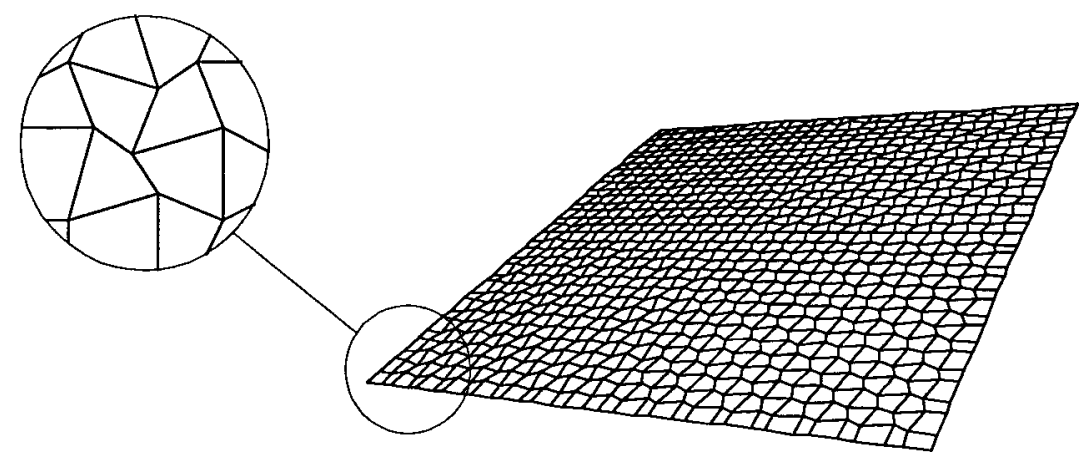

Figure 10. Distorted mesh of the square plate 


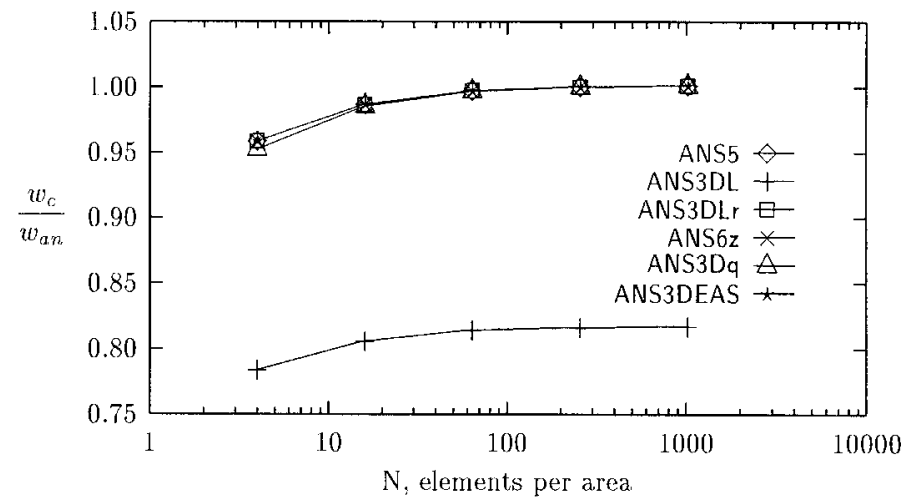

Figure 11. Convergence investigation for various element formulations; thin square plate with distorted mesh

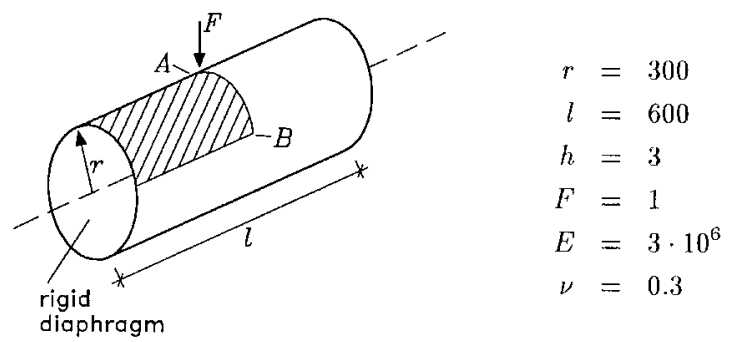

Figure 12. Geometry and material data of the pinched cylinder, see e.g. Reference 16

5.2.2. Pinched cylinder subjected to opposite point loads. This highly curved structure as given in Figure 12 is known to cause a low convergence rate especially in the case of elements with linear shape functions. For the following analyses only an eighth of the cylinder is discretizied due to symmetry, which is then also enforced on the results. The displacements of the upper and lower surface are computed for the loading point, but due to the minor differences of both displacements only the displacements of the upper surface are shown here.

In this problem it is not possible to match the boundary conditions of the degenerated shells correctly by using a discretization with one element in thickness direction for the solid-shells and therefore some differences are found, see Figure 13. Although the vertical displacements of the proposed elements differ from the ANS5 shell element clearly for coarse meshes, they coincide quite well in the case of finer meshes when the number of elements reaches the number necessary to get results close to the analytical solution also for the ANS5 shell element. Theses differences for coarse meshes can be attributed to the fact that the boundary condition at the longitudinal edge, which is different for the solid-shell and the shell element, is felt more for too coarse meshes. Again, the ANS3DL element locks slightly.

Additional linear investigations not presented here for brevity reasons show that the ANS3Dq, ANS3DLr and ANS3DEAS elements are able to describe transversal load conditions correctly. As expected the ANS3DL elements behaves too stiff due to locking and the ANS6z element behaves too flexible due to the neglected coupling terms. 


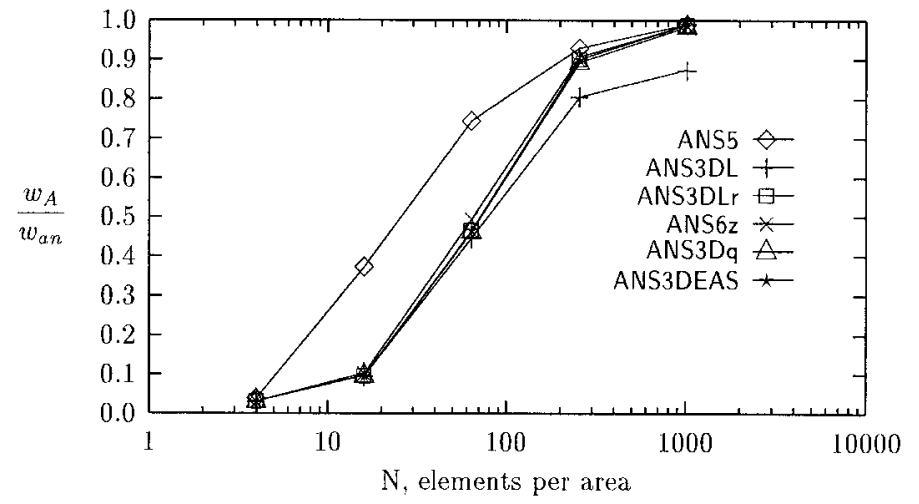

Figure 13. Convergence investigation for the pinched cylinder; displacements of upper surface

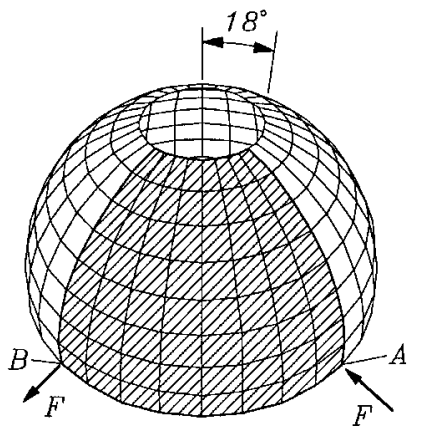

$$
\begin{aligned}
r & =10 \\
h & =0.01 \\
E & =6.825 \cdot 10^{7} \\
\nu & =0.3 \\
F & =2.0
\end{aligned}
$$

Figure 14. Geometry and material data of the hemisphere

\subsection{Geometrically non-linear analyses}

5.3.1. Hemisphere loaded by pinching forces. A hemisphere with a $18^{\circ}$ hole loaded by pinching forces is often cited as a benchmark problem for shell elements to analyse the ability to model rigid body modes and inextensional bending. To obtain comparable results to investigations by Parisch $^{12}$ the same geometry and material data (see Figure 14) and the same coarse discretization with $16 \times 16 \times 1$ bilinear solid-shell elements per quarter section are used.

As expected the load deflection curves indicate in the case of larger displacements for the solid-shell elements a stiffer behaviour than for the 4-node degenerated shell element (ANS5), see Figure 15. There are no differences between the load deflection curves obtained with the ANS3Dq element and between the load deflection curve of the 3D-SHEL4 element presented in Reference 12. The reason is that the pinched hemisphere is not dominated by transversal shear and thus the director remains normal to the mid-surface even in the deformed configuration.

However, additional investigations show that a discretization with $16 \times 16 \times 1$ elements per quarter section is far too coarse to get reliable results. Thus, the load deflection curves presented in Figure 16 are computed using a refined mesh with $32 \times 32 \times 1$ elements. 


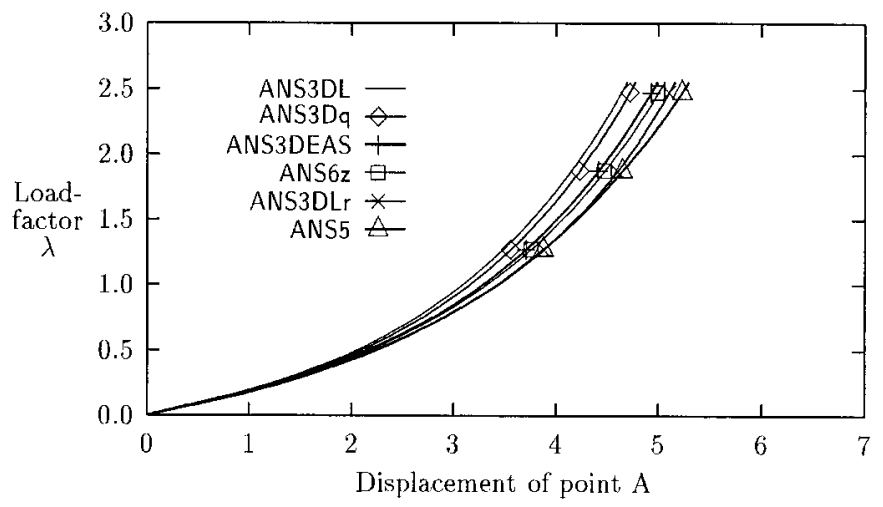

Figure 15. Pinched hemisphere; $16 \times 16 \times 1$ elements; load-deflection diagram; displacement of point A

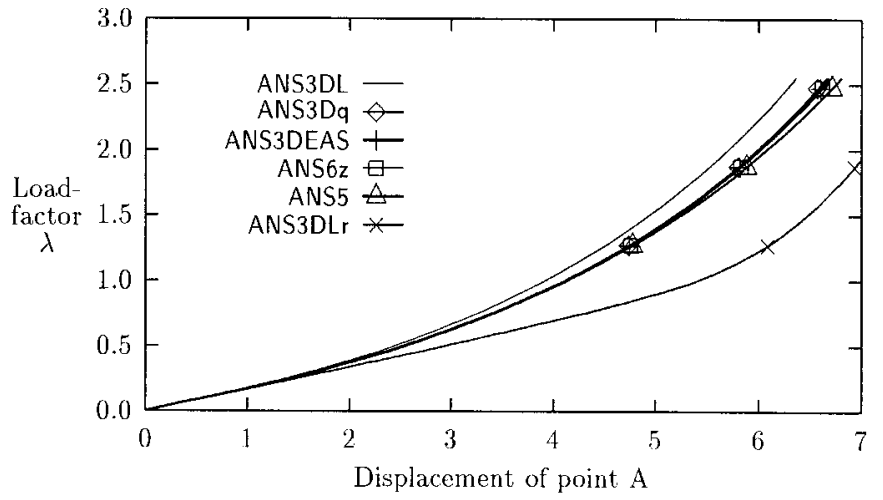

Figure 16. Pinched hemisphere; $32 \times 32 \times 1$ elements; load-deflection diagram; displacement of point A

Then even for larger displacements there are no significant differences visible in the load deflection curves of the degenerated shell element and of the solid-shell elements ANS6z, ANS3Dq and ANS3DEAS. In equivalence to the previous linear analyses the ANS3DL element behaves too stiff and the ANS3DLr element behaves too flexible.

5.3.2. Pinched cylinder subjected to opposite point loads. For the geometry and material data the same values as given in Figure 12 are used. Due to the symmetry of the structure, only one eighth of the cylinder is investigated using on purpose a fairly coarse mesh with $24 \times 24$ elements in in-plane and one element in thickness direction. Although it is impossible to match the boundary conditions of the degenerated shell elements correctly, the difference in the load displacement curves of the ANS6z, ANS3Dq, ANS3DEAS elements and the ANS5 element are not significant which is particulary visible for smaller displacements. However, when the displacements increase the difference in the boundary conditions of the diaphragm is responsible for the larger difference in the results. As already obtained in Section 2.1.2, the ANS3DL element locks which is visible already for rather small displacements and thus the difference to the other load-deflection curves 


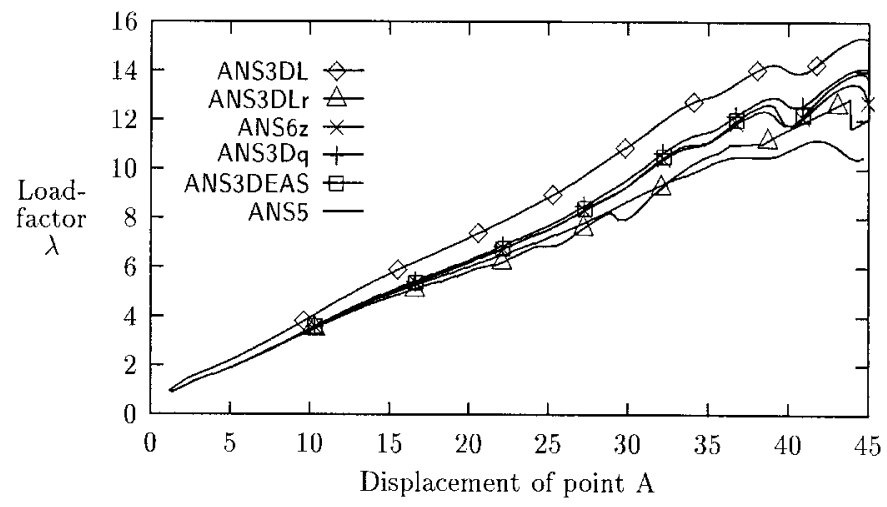

Figure 17. Pinched cylinder; load-deflection diagram; displacement of point A

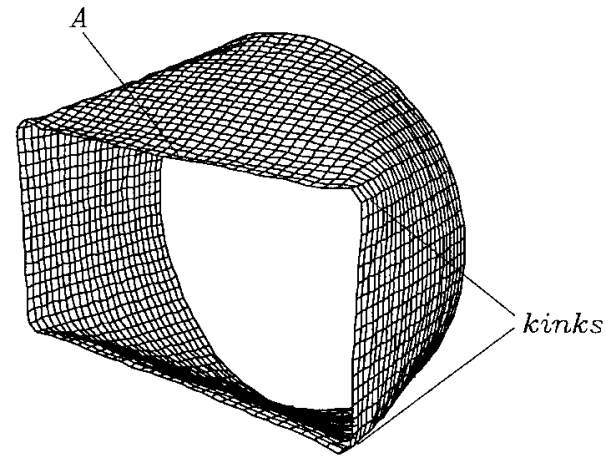

Figure 18. Deformed geometry of the pinched cylinder at the last load increment

becomes larger with increasing displacements. Again the ANS3DLr element shows clearly a too flexible behaviour due to the missing coupling terms. In addition, convergence is not obtained throughout the whole load displacement curve because the mesh is too coarse, but total convergence can be achieved using a finer mesh.

Thus, it can be stated that the ANS3DLr element is not as robust as the ANS6z, ANS3Dq or ANS3DEAS elements in the non-linear analysis of highly curved structures.

It also must be mentioned that the 'non-smooth' curves observed in the load deflection diagrams for larger displacements apparently indicating a snap through like behaviour are a result of the rather coarse mesh taken here on purpose for all elements to investigate the robustness. The wriggles in the curves occur because kinks are developing during the loading process (see Figure 18) which cannot be correctly described by coarse meshes. They disappear completely for finer meshes, which are not shown here for brevity reasons.

\section{CONCLUSIONS}

In the present contribution an alternative formulation to the degenerated shell concept is presented. The proposed so-called solid-shell concept incorporates only displacement degrees of freedom 
and is based on a linear approximation of all displacements in thickness direction, whereas the approximation in in-plane direction can be enhanced easily. Thus, the assumption of the standard shell concepts that the normals remain straight is fulfilled. Due to the absence of rotational degrees of freedom, the need for special co-ordinate systems at boundaries and, in geometrically non-linear analyses, the complicated update of the rotation vector is avoided. Also the boundary conditions can be enforced in a physically clear manner, as only displacements are involved. Contrary to the degenerated shell concept normal stresses and strains in thickness direction can be evaluated. In addition, no special transition elements are necessary, if solid-shell elements are combined with solid elements. However, there is a drawback of a minor increase of the total number of degrees of freedom.

Several locking-free solid-shell element formulations are discussed. Inspecting an element that is based on the solid-shell concept without any further modification locking appears due to the coupling of the membrane strains and the normal strains in thickness direction. Two ways to avoid this locking phenomena are proposed. The first one is based on the introduction of the plane stress condition and the second one on the introduction of linear strains in thickness direction in a direct way leading to two locking-free solid-shell element formulations for each approach. The major disadvantage of the two solid-shell elements based on the plane stress condition is the limitation to reduced material laws whereas the both elements based on the direct assumption of a linear strain distribution in thickness direction are less efficient. However, the overall behaviour of all proposed locking-free solid-shell elements is very favourable.

Obviously, it is not possible using the proposed solid-shell element formulations to describe three-dimensional stress states correctly by using a discretization with only one element through the thickness. Although with the application of more elements in thickness direction this limitation could be overcome, the mesh input becomes rather complicated. It is then preferable to apply higher-order approximations of all displacements in thickness direction-in-plane displacements $u^{\prime}$ and $v^{\prime}$ at least cubic and the displacement in thickness direction $w^{\prime}$ at least quadratic (see e.g. References 6 and 7).

However, due to the possibility to combine the proposed element formulations straightforward with standard solid elements, there seems to be no need for the latter approach.

\section{REFERENCES}

1. S. Ahmad, B. M. Irons and O. C. Zienkiewicz, 'Analysis of thick and thin shell structures by curved finite elements', Int. J. Numer. Meth. Engng., 2, 419-451 (1970).

2. K. J. Bathe, Finite Element Procedures in Engineering Analysis, Prentice-Hall, Englewood Cliffs, N.J., 1982.

3. E. Ramm, 'A plate/shell element for large deflections and rotations', in K. J. Bathe et al., Formulations and Computational Algorithms in Finite Element Analysis, MIT Press, Cambridge, MA, 1977.

4. R. D. Mindlin, 'Influence of rotatory inertia and shear in flexural motions of isotropic elastic plates', J. Appl. Mech. 18, 31-38 (1951).

5. E. Reissner, 'The effect of transverse shear deformation on the bending of elastic plates', J. Appl. Mech., 12, 69-76 (1945).

6. C. Sansour, 'A theory and finite element formulation of shells at finite deformations involving thickness change', Arch. Appl. Mech., 65, 194-216 (1995).

7. M. Braun, Nichtlineare Analysen von geschichteten elastischen Flächentragwerken, Bericht Nr. 19, Institut für Baustatik, Universität Stuttgart, 1995.

8. F. Gruttmann, Theorie und Numerik dünnwandiger Faserverbundstrukturen, Bericht No. F96/1, Institut für Baumechanik und Numerische Mechanik, Universität Hannover, 1996.

9. J. O. Hallquist, Latest developments in LS-DYNA3D vs 910, Livermore Software Technology Corporation, 1991.

10. K. J. Bathe and E. Dvorkin, 'A continuum mechanics based four-node shell element for general nonlinear analysis', Engng. Comput., 1, 77-88 (1984). 
11. B. Seifert, Zur Theorie und Numerik Finiter elastoplastischer Deformationen von Schalenstrukturen, Bericht No. F96/2, Institut für Baumechanik und Numerische Mechanik, Universität Hannover, 1996.

12. H. Parisch, 'A continuum-based shell theory for non-linear applications', Int. J. Numer. Meth. Engng., 38, 1855-1883 (1995).

13. H. Schoop, 'Oberflächenorientierte Schalentheorien endlicher Verschiebungen', Ingenieur-Arch., 56, $427-437$ (1986).

14. N. Büchter and E. Ramm, '3D-extension of nonlinear shell equations based on the enhanced assumed strain concept', in Ch. Hirsch, J. Periaux and E. Onate (eds.), Computational Methods in Applied Sciences, Amsterdam, 1992.

15. J. C. Simo and M. S, Rifai, 'A class of mixed assumed strain methods and the method of incompatible modes', Int. J. Numer. Meth. Engng., 29, 1595-1638 (1990).

16. T. Belytschko, H. Stolarski, W. K. Liu, N. Carpenter and J. S. J. Ong, 'Stress projection for membrane and shear locking in shell Finite Elements', Comput. Meth. Appl. Mech. Engng., 51, 221-258 (1985).

17. R. Hauptmann, Entwicklung und Gegenüberstellung von strukturangepä̈ten, geometrisch nichlinearen Finiten Elementen zur effizienten Flächentragwerksberechnung, Institut für Mechanik, Universität Karlsruhe, Dissertation, 1997, submitted.

18. H. Gebhardt, Finite Element Konzepte für schubelastische Schalen mit endlichen Drehungen, Dissertation, Institut für Baustatik, Universität Karlsruhe, 1990.

19. S. Timoshenko and S. Woinowsky-Krieger, Theory of Plates and Shells, 2nd. edn, McGraw-Hill, New York, 1959.

20. M. A. Crisfield, Non-linear Finite Element Analysis of Solids and Structures, Wiley, Chichester, New York, Brisbane, Toronto, Singapore, 1991.

21. A. C. Scordelis and K. S. Lo, 'Computer Analysis of Cylindrical Shells', J. Amer. Concr. Inst., 61, $539-561$ (1969). 